\title{
METHES: A Monte Carlo collision code for the simulation of electron transport in low temperature plasmas
}

Journal Article

Author(s):

Rabie, Mohamed; Franck, Christian (1)

Publication date:

2016-06

Permanent link:

https://doi.org/10.3929/ethz-a-010881688

Rights / license:

Creative Commons Attribution-NonCommercial-NoDerivatives 4.0 International

Originally published in:

Computer Physics Communications 203, https://doi.org/10.1016/j.cpc.2016.02.022 
This is a post-refereeing copy of the manuscript submitted to Computer Physics Communications.

The original version can be found on the Computer Physics Communications website:

Digital Object Identifier: http://dx.doi.org/10.1016/j.cpc.2016.02.022

(C) 2016. This manuscript version is made available under the CC-BY-NC-ND 4.0 license http://creativecommons.org/licenses/by-nc-nd/4.0/ 


\title{
METHES: A Monte Carlo collision code for the simulation of electron transport in low temperature plasmas
}

\author{
M. Rabie ${ }^{1}$, C.M. Franck
}

Power Systems and High Voltage Laboratories, ETH Zurich, 8092 Zurich, Switzerland

\begin{abstract}
We present a freely available MATLAB code for the simulation of electron transport in arbitrary gas mixtures in the presence of uniform electric fields. For steady-state electron transport, the program provides the transport coefficients, reaction rates and the electron energy distribution function. The program uses established Monte Carlo techniques and is compatible with the electron scattering cross section files from the open-access Plasma Data Exchange Project LXCat. The code is written in object-oriented design, allowing the tracing and visualization of the spatiotemporal evolution of electron swarms and the temporal development of the mean energy and the electron number due to attachment and/or ionization processes. We benchmark our code with well-known model gases as well as the real gases argon, $\mathrm{N}_{2}, \mathrm{O}_{2}$, $\mathrm{CF}_{4}, \mathrm{SF}_{6}$ and mixtures of $\mathrm{N}_{2}$ and $\mathrm{O}_{2}$.
\end{abstract}

Keywords: Low temperature plasmas, Electron transport PACS: 52.65.Pp, Monte Carlo methods PACS: 52.20.Fs, Electron collisions PACS: 52.25.Fi, Transport properties

\section{PROGRAM SUMMARY}

Manuscript Title: METHES: A Monte Carlo collision code for the simulation of electron transport in low temperature plasmas Authors: Mohamed Rabie, Christian M. Franck

Email address: rm@ethz.ch (M. Rabie)

${ }^{1}$ phone: +41446328430 
Program Title: METHES

Journal Reference:

Catalogue identifier:

Licensing provisions: none

Programming language: MATLAB R2014 (very likely compatible with earlier versions)

Computer: any which supports MATLAB

Operating system: any which supports MATLAB

$R A M$ : variable, depending on number of electrons

Keywords: Monte Carlo methods, Electron collisions, Transport properties Classification: 19.11

Nature of problem: Simulation of electron transport in arbitrary gas mixtures in the presence of electric fields.

Solution method: Monte Carlo Method.

Running time: depending on computer and desired precision of results between several minutes and several hours.

\section{Introduction}

Electron transport in a gas subject to electric fields is crucial for various applications such as gas based radiation detectors [1, 2], plasma processing [3] and high-voltage insulation [4]. Simulations support the understanding of the fundamental processes but are also technologically relevant for the design of equipment. The kinetic modeling on the basis of microscopic collision cross sections is possible either by numerically solving the Boltzmann equation for the electron distribution [5-7], or by tracking the stochastic motion of the individual electrons by means of Monte Carlo simulation [1, 8-12]. For steady-state conditions kinetic modeling can provide transport parameters and reaction rates that are the necessary input parameters for macroscopic fluid models used in low-temperature plasmas and gas discharges [13].

Both Boltzmann solvers and Monte Carlo methods require the knowledge of all relevant types of electron-neutral collisions that are possible in a gas or gas mixture. The most common methods to obtain the corresponding cross sections for individual processes are ab-initio quantum theoretical calculations [14], electron beam measurements [15] and electron swarm experiments [16]. The latter provide cross sections indirectly from transport parameters and reaction rates. The main challenge is to collect, compare and share the 
cross section data and decide which data set to use for a certain species. This procedure has been greatly simplified by the open-access Plasma Data Exchange Project LXCat [17], which provides in addition to cross section data also transport parameters and reaction rates as a function of the reduced electric field $E / N$, which is the ratio of the electric field strength to the gas particle number density.

Several Boltzmann solvers and Monte Carlo programs are only commercially available or are inconvenient in sense of data handling or are suffering from limited source information. The freely available Boltzmann solver BOLSIG $+[7]$ provides a user-friendly and fast option to calculate transport parameters and reaction rates and is compatible with the cross section data from LXCat. However, BOLSIG+ is based on the two-term approximation, whose applicability has been discussed in the literature $[18,19]$. The precision of Monte Carlo methods depends, when no numerical integrations are used, mainly on the precision of the used cross sections. The disadvantage of Monte Carlo methods is the long run-time compared to Boltzmann solvers when reasonable precision is needed. A freely-available Monte Carlo program is MAGBOLTZ [1]. The cross sections for this program are embedded in the code and straightforward comparison with other programs such as BOLSIG+ is therefore complicated. Therefore, it is often unclear whether the source of possible discrepancies between the results of BOLSIG+ and MAGBOLTZ arise from different cross section sets or from numerical approximations such as the two-term approximation. Furthermore, BOLSIG+ and MAGBOLTZ do not provide bulk transport data, whereas the distinction between flux and bulk transport parameters might be important for plasma modeling and the derivation of cross sections from measured transport data [19-22].

In this work, we present a user-friendly Monte Carlo program that calculates flux and bulk transport parameters, reaction rates and electron energy distribution functions (EEDF) for arbitrary gas mixtures. Cross section sets complying with the format of LXCat are needed as an input. Thus, crosschecking the results of e.g. BOLSIG + and the present program is possible and confusion about different cross section inputs as a possible source of discrepancies between the results of both programs can be eliminated. The code is written object-orientated in MATLAB, allowing a single-core simulation (@ $2.5 \mathrm{GHz} \mathrm{CPU}$ ) of up to $10^{6}$ electrons simultaneously. Furthermore, all functions are well documented so that modification or extension of the code by the user is possible. For non-steady state processes the temporal development of an electron avalanche can be investigated in-situ and visualized 
in terms of electron trajectories, electron densities, electron number, mean energy and mean position.

We test our program using the Maxwell model [23] and Reid's ramp model [24]. An extensive test of the code is performed in argon over a wide $E / N$ range using various numerical settings. In order to directly compare our results with those of BOLSIG+ and MAGBOLTZ, we calculate the mean energy, transport data and reaction rates in argon, $\mathrm{N}_{2}$ and $\mathrm{O}_{2}$. In addition, we apply our code for different $\mathrm{N}_{2} / \mathrm{O}_{2}$ mixtures as well as to the electron attaching gases tetrafluoromethane $\mathrm{CF}_{4}$ and sulfur hexafluoride $\mathrm{SF}_{6}$.

\section{Methods}

In this section we describe the methods for modeling electron trajectories in the presence of collisions with a background gas in a uniform electric field. Figure 1 gives an overview of the structure of METHES. The input data of electron-molecule collision cross sections has the form provided by the LXCat database. The class "ImportLXCat" imports the cross sections and the corresponding threshold energies for ionization and excitation processes. The details are described in section 2.1. Subsequently, the class "MonteCarlo" contains all methods and properties that are necessary for the Monte Carlo simulation. In section 2.2, the principles of determining the collision times and the collision types are briefly summarized in case of a single electron. The matrix formalism that is needed for following the trajectories of an electron ensemble is described in section 2.3. The treatment of the collision processes in sections 2.4 and 2.5 is followed by the description of the sample techniques that allow to extract the energy data, transport parameters and reaction rates. MATLAB has a built-in feature for documentation of classes, methods and properties. Typing e.g. doc MonteCarlo" into the MATLAB command window gives a description of the class MonteCarlo and information on the methods and the properties.

\subsection{Cross sections}

The relevant types of electron-neutral collisions for the Monte Carlo simulation are elastic, inelastic, ionization and attachment collisions. The collision data for a specific species can be either downloaded from the LXCat database, or be constructed by the user conserving the format of LXCat. "LXCat uses the key words "elastic" and "momentum" to refer to "elastic 


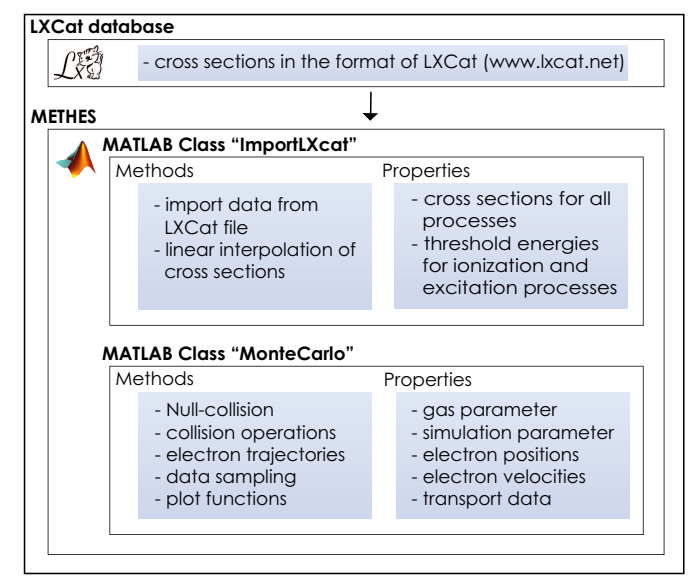

Figure 1: Overview of the METHES code

momentum transfer" and "effective momentum transfer" cross sections, respectively. A better choice of keywords would be "elastic" and "effective", and we (comment: Pancheshnyi et al.) intend to phase in this change" [17]. If the "effective momentum transfer" cross section is given, then the code calculates the "elastic momentum transfer" cross section by subtracting all inelastic cross sections (keyword "excitation"), including ionization. This "elastic momentum transfer" cross section is then used together with the inelastic, ionization and attachment cross sections (keywords "ionization" and "attachment", respectively) for the simulation. A linear interpolation is used between the points in a table of cross section versus energy. Below the first data point and beyond the last data point, METHES assumes that the cross section is constant at the values of these data points. No differential cross sections are used by our method.

\subsection{Null-collision technique}

The times between collisions can be determined either by the integration technique [25] or the null-collision technique [8]. In our code the latter is used, which allows to choose times between collisions, without any approximation or numerical integration by the use of a constant trial collision frequency $\nu^{\prime}$ that for all times must be larger than the total collision frequency

$$
\nu^{\prime}>\max \left(n \sigma_{\mathrm{T}} v\right) .
$$

Here, $n$ is the space- and time-independent total gas number density, $v(t)$ is the electron velocity and $\sigma_{\mathrm{T}}(\varepsilon)$ is the total collision cross section. For a gas 
mixture of $K$ components with partial fraction $k_{i}$ of the $i^{\text {th }}$ gas species, the total cross section is given by

$$
\sigma_{\mathrm{T}}=\sum_{i=1}^{K} \sum_{j=1}^{S_{i}} k_{i} \sigma_{i, j} .
$$

The $i^{\text {th }}$ gas species has $S_{i}$ different types of collisions with the cross section $\sigma_{i, j}$ for the $j^{\text {th }}$ type, where $1 \leq j \leq S_{i}$.

The time between collisions depends on $\nu^{\prime}$ and on the random numbers $p_{\mathrm{c}}$ in the interval $[0,1]$ and is given by

$$
\Delta t=-\frac{1}{\nu^{\prime}} \ln \left(p_{\mathrm{c}}\right) .
$$

The time of collision events is assumed to be much smaller than the time between collisions and therefore is set to zero. The thermal energy of the background gas is neglected at this point, which is a good approximation at moderate and high $E / N$ values but is known to affect the electron transport parameters at low $E / N$ values when the kinetic energy of electron and gas molecules are of the same order of magnitude [10].

\subsection{The null-collision technique for an electron ensemble}

During the time $\Delta t$ the $N_{\mathrm{e}}$ electrons gain energy in the electric field $\mathbf{E}$. The positions $\mathbf{r}$ and velocities $\mathbf{v}$ at each time are followed. In Cartesian coordinates $\mathbf{r}$ and $\mathbf{v}$ are of the form

$$
\mathbf{r}=\left(\begin{array}{ccc}
r_{x}^{(1)} & r_{y}^{(1)} & r_{z}^{(1)} \\
r_{x}^{(2)} & r_{y}^{(2)} & r_{z}^{(2)} \\
\vdots & \vdots & \vdots \\
r_{x}^{\left(N_{\mathrm{e}}\right)} & r_{y}^{\left(N_{\mathrm{e}}\right)} & r_{z}^{\left(N_{\mathrm{e}}\right)}
\end{array}\right), \mathbf{v}=\left(\begin{array}{ccc}
v_{x}^{(1)} & v_{y}^{(1)} & v_{z}^{(1)} \\
v_{x}^{(2)} & v_{y}^{(2)} & v_{z}^{(2)} \\
\vdots & \vdots & \vdots \\
v_{x}^{\left(N_{\mathrm{e}}\right)} & v_{y}^{\left(N_{\mathrm{e}}\right)} & v_{z}^{\left(N_{\mathrm{e}}\right)}
\end{array}\right)
$$

For the derivation of the transport parameters, a uniform field in z-direction of amplitude $E_{\mathrm{z}}$ is considered

$$
\mathbf{E}=\left(\begin{array}{ccc}
0 & 0 & E_{z} \\
0 & 0 & E_{z} \\
\vdots & \vdots & \vdots \\
0 & 0 & E_{z}
\end{array}\right)
$$


During the time $\Delta \mathrm{t}$, the electron position $\mathbf{r}$ and velocity $\mathbf{v}$ undergo the transformation

$$
\begin{aligned}
& \mathbf{r} \longrightarrow \mathbf{r}+\mathbf{v} \Delta t-0.5 e \mathbf{E} / m_{\mathrm{e}} \Delta t^{2} \\
& \mathbf{v} \longrightarrow \mathbf{v}-e \mathbf{E} / m_{\mathrm{e}} \Delta t
\end{aligned}
$$

Here $e$ and $m_{\mathrm{e}}$ are the electron charge and the electron mass.

After a period $\Delta t$ of free motion, the decision has to be made which electron undergoes which type of collision. For the $k^{\text {th }}$ electron the collision frequency for each process is recorded. Since the cross section for a process depends on the electron energy, we define the norm of $\mathbf{v}$ as a $N_{\mathrm{e}} \times 1$ vector $|\mathbf{v}|$ with the entries

$$
|\mathbf{v}|_{\mathbf{k}}=\sqrt{\sum_{d=x, y, z}\left(\mathbf{v}_{d}^{(k)}\right)^{2}} .
$$

The corresponding kinetic energies of the electrons are given in the vector $\epsilon$ with the entries

$$
\epsilon_{\mathbf{k}}=m_{\mathrm{e}}\left|\mathbf{v}_{\mathbf{k}}\right|^{2} / 2
$$

We construct a $N_{\mathrm{e}} \times M$ matrix, which contains the collision frequencies of all possible processes column-wise, where $M=\sum_{i=1}^{K} S_{i}$. Subsequently the matrix is normalized with the collision frequency $\nu^{\prime}$, and the rows are summed up cumulatively to obtain the (column-wise) monotonically increasing Matrix $\mathrm{C}$, which can be expressed as

$$
\begin{aligned}
C_{1 \ldots N, m} & =\frac{n|\mathbf{v}|}{\nu^{\prime}} \sum_{i=1}^{f(m)} \sum_{j=1}^{g(i, m)} k_{i} \sigma_{i, j} \\
f(m) & =1+\sum_{m^{\prime}=1}^{m} \sum_{i=1}^{K} \delta_{m^{\prime}, \sum_{i^{\prime}=1}^{i} S_{i^{\prime}}} \\
g(i, m) & =m+S_{i}-\sum_{i^{\prime}=1}^{i} S_{i^{\prime}},
\end{aligned}
$$

with the Kronecker-Delta $\delta$.

Finally, a $N_{\mathrm{e}} \times 1$ vector $\mathbf{p}$ of random numbers with entries in the interval $[0,1]$ is introduced, which is refreshed at each time step. Each line in $\mathrm{C}$ is compared to the same line in $\mathbf{p}$ : if the random number is the smallest number, then the first collision process occurs. If the random number is 
larger than the $(m-1)^{\text {th }}$ and smaller than the $m^{\text {th }}$ entry in the line of $\mathrm{C}$, then the collision process $m$ happens. In case of the random number being the largest number no collision occurs. This is called a null-collision.

\subsection{Conservative collisions}

From the previous section it is known which electron will undergo which type of collision after the time step $\Delta t$. Here, we give the details of how conservative collisions are treated within the code. These are the collisions in which the number of electrons does not change, namely elastic and inelastic collisions. In case of a null-collision the electron velocity after the collision remains unchanged, whereas for elastic or inelastic collisions the electron is isotropically scattered. The change of direction is given by the azimuthal $\phi$ and polar angle $\theta$, which are determined by the random numbers $p_{1}$ and $p_{2}$ in the interval $[0,1]$ via the relations

$$
\begin{aligned}
\phi & =2 \pi p_{1}, \\
\cos (\theta) & =1-2 p_{2} .
\end{aligned}
$$

The energy loss due to an elastic collision with a gas particle of mass $M$ is given by

$$
\Delta \varepsilon=\varepsilon \frac{2 m_{\mathrm{e}}}{M}(1-\cos \theta),
$$

whereas for an inelastic collision the energy-loss corresponds to the threshold energy $\varepsilon^{*}$ of a certain electronic, vibrational or rotational excitation

$$
\Delta \varepsilon=\varepsilon^{*} .
$$

\subsection{Non-conservative collisions}

In our code we consider ionization and attachment as non-conservative processes, but no other electron number changing processes such as detachment or recombination. Two different ways to model non-conservative collisions are provided by the code:

- Non-fixed electron number $N_{\mathrm{e}}(t)$ : the electrons that are created in ionization events and annihilated during attachment events are taken into account during the simulation. In an ionization event an additional electron is created at the same position and the remaining energy is shared with a factor $0 \leq W \leq 1$, where e.g. $W=0.5$ would correspond to equal energy sharing between the electrons. Similar to the 
case of conservative collisions, the direction of the velocity vector for both electrons is given by the random scattering angles from equations (13)-(14). In an attachment process the electron is simply removed from the electron ensemble. The uncertainty of the results strongly increases in case of strong attachment or ionization: for strong ionization the exponential increase of the electron number will reach the limit set by the memory resource before enough collisions are performed to extract accurate transport parameters. Similarly, in the limit of strong attachment, most electrons might be attached before the steady state is reached, precluding the extraction of transport data due to a too small ensemble size.

- Fixed electron number $N_{\mathrm{e}}(t)=N_{\mathrm{e}}$ : the number of electrons is fixed to a constant value even in case of strong ionization or attachment. We use a technique that does not affect the average properties of the electron ensemble, see e.g. [26]: in case of an ionization event, an electron is created and energy is shared between electrons with the factor $W$. Subsequently a random electron is removed from the ensemble in order to keep the electron number constant. Similarly, for an attachment event the electron is removed and then a random electron from the remaining ensemble is cloned in terms of position and energy. In contrast to the non-fixed method, within the fixed method the transport data can be obtained even for strong attachment or ionization.

\subsection{Data sampling}

The mean energy, electron energy distribution, transport coefficients and reaction rates are determined from the electron's kinetic energies $\varepsilon$, positions $\mathbf{r}$, velocities $\mathbf{v}$ and the numbers of reaction events, after the electrons reached the steady-state. This time $t_{0}$ strongly depends on the gas species and on $E / N$ and is automatically determined for each simulation from the evolution of the electron ensemble's mean energy, as illustrated in figure 2: as soon as the mean energy remains beside statistical noise temporally constant, the system is considered to be in the steady-state. The most relevant parameters such as the electron energy probability function, mean energy, position and width of the electron ensemble are plotted during the simulation. The user can monitor those parameters to check if the steady-state has been reached. 

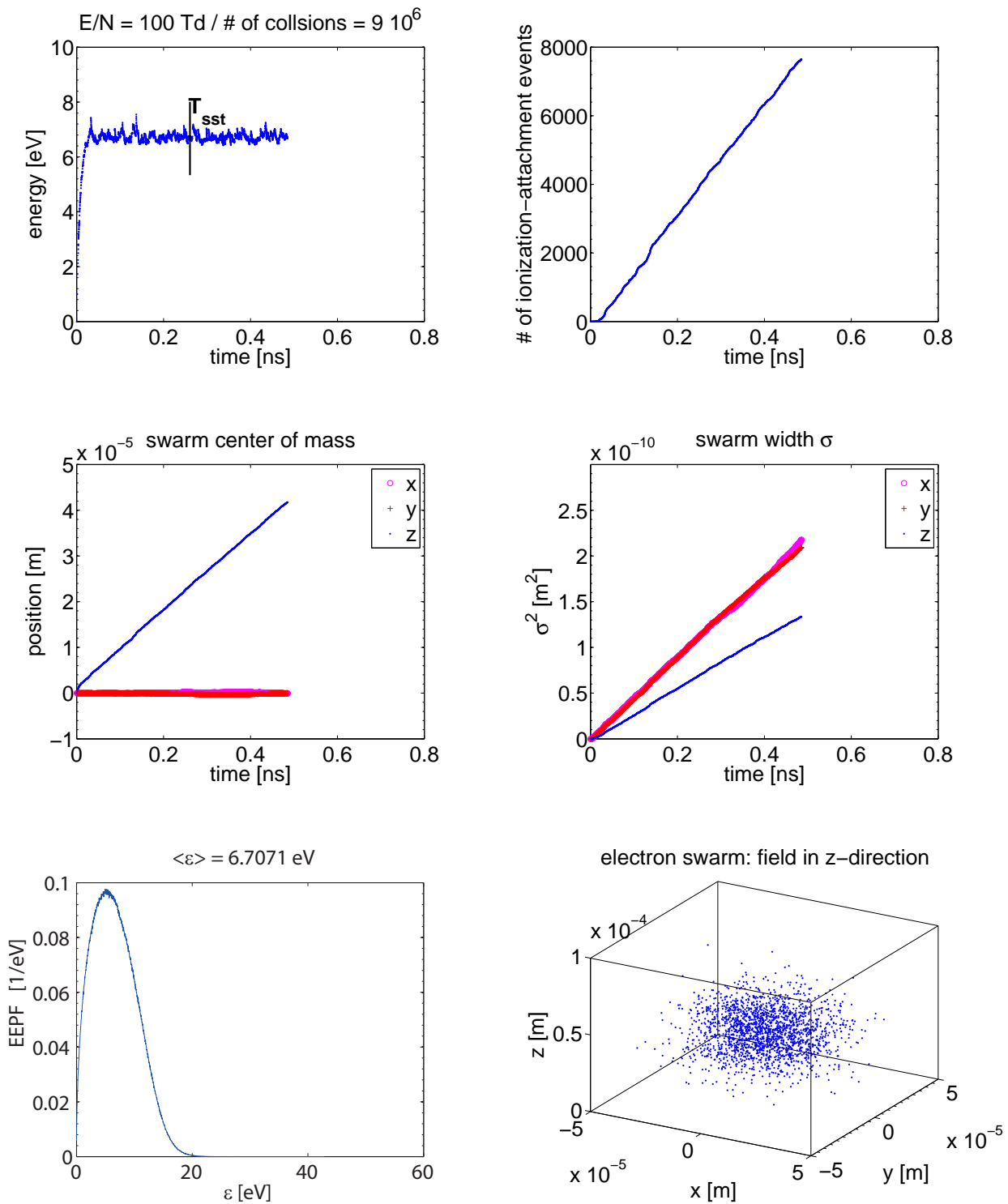

Figure 2: A screenshot during the METHES simulation showing selected swarm properties vs time. Settings: argon, electric field into z-direction, $E / N=100 \mathrm{Td}, p=10^{5} \mathrm{~Pa}$, energy sharing $W=0.5$ and electron number fixed to $N_{\mathrm{e}}=10^{4}$. The energy and position of the initial electrons have been set to zero. Upper left: mean energy $\langle\varepsilon(t)\rangle$ at current point in time. After the indicated point of time $T_{\mathrm{sst}}=t_{0}$ the swarm is considered to be in steady-state and the swarm data is being collected. Upper right: number of ionization events $N_{i^{-}}$number of attachment events $N_{a}$. The slope of the curve is proportional to $\nu_{\text {eff }}$, as describled by equation (27). Middle left: $\langle\mathbf{r}\rangle$ in $\mathrm{x}-, \mathrm{y}-$ and $\mathrm{z}-$ direction. The slopes of the curves are equal to $\mathbf{w}$, according to equation (20). Middle right: $\left\langle(\mathbf{r}-\langle\mathbf{r}\rangle)^{2}\right\rangle$ in $\mathrm{x}^{-}, \mathrm{y}-$ and $\mathrm{z}$ - direction. The slopes of the curves are equal to $2 \cdot \mathbf{D}$, according to equation (21). Lower left: EEPF collected from $t_{0}$ until current point in time. Lower right: electron distribution in space at current point in time. 


\subsubsection{Mean energy and electron energy distribution}

The mean energy $\langle\varepsilon\rangle$ is derived by time-averaging the energies of all electron trajectories

$$
\langle\varepsilon\rangle=\frac{1}{T} \frac{m_{\mathrm{e}}}{2} \sum_{k=1}^{N_{\mathrm{e}}} \int_{t_{0}}^{t_{\mathrm{f}}}\left|\mathbf{v}_{\mathbf{k}}(t)\right|^{2} d t,
$$

whereas the time $T$ is the sum of the travel times of all electrons between $t_{0}$ and the time at the end of the simulation $t_{\mathrm{f}}$. The integral in equation (17) is analytically calculated between collisions using the right hand side of equation (7).

The electron energy probability function (EEPF) $p(\varepsilon)$ is derived from counting the number of electron energies within energy increments $d \varepsilon$. The code ensures no cutoff of high-energy electrons and automatically determines an upper bound for the energy until which the EEPF is sampled. The number of energy intervals, and thus indirectly the size of $d \varepsilon$, can be specified by the user. Before steady-state is reached, the program extracts the EEPF for each point in time. For these "snap-shots", the noise is relatively high due to the small number of sampling data, which corresponds to the used electron number for the simulation. As soon as the electrons are in steady-state, the $\mathrm{EEPF}$ is averaged over the interval $\left[t_{0}, t_{\mathrm{f}}\right]$ and the accuracy of the EEPF increases with time.

The probability function EEPF is normalized

$$
\int_{0}^{\infty} p(\varepsilon) d \varepsilon=1
$$

whereas the electron energy distribution function (EEDF) $f(\varepsilon)$ is calculated by

$$
f(\varepsilon)=\varepsilon^{-1 / 2} p(\varepsilon) .
$$

\subsubsection{Transport coefficients}

The bulk drift velocity $w$ and diffusion constant $D[27]$

$$
\begin{aligned}
\mathbf{w} & =\frac{d}{d t}\langle\mathbf{r}\rangle, \\
\mathbf{D} & =\frac{1}{2} \frac{d}{d t}\left\langle(\mathbf{r}-\langle\mathbf{r}\rangle)^{2}\right\rangle,
\end{aligned}
$$


are obtained as the coefficient estimates for a linear regression of the swarm's center of mass $\langle\mathbf{r}\rangle$, and of the squared width $\left\langle(\mathbf{r}-\langle\mathbf{r}\rangle)^{2}\right\rangle$ respectively, versus time $t-t_{0}$.

The flux drift velocity $\tilde{w}$ and diffusion constant $\tilde{D}[28]$

$$
\begin{aligned}
\tilde{\mathbf{w}} & =\left\langle\frac{d r}{d t}\right\rangle, \\
\tilde{\mathbf{D}} & =\langle r v\rangle-\langle r\rangle\langle v\rangle .
\end{aligned}
$$

are determined, similar as for the derivation of $\langle\varepsilon\rangle$, as average over all electron trajectories

$$
\begin{aligned}
\tilde{\mathbf{w}} & =\frac{1}{T} \sum_{k=1}^{N_{\mathrm{e}}} \int_{t_{0}}^{t_{\mathrm{f}}} \mathbf{v}_{\mathbf{k}}(t) d t \\
\tilde{\mathbf{D}} & =\sum_{k=1}^{N_{\mathrm{e}}}\left(\frac{1}{T} \int_{t_{0}}^{t_{\mathrm{f}}} \mathbf{r}_{\mathbf{k}}(t) \mathbf{v}_{\mathbf{k}}(t) d t-\frac{1}{T} \int_{t_{0}}^{t_{\mathrm{f}}} \mathbf{r}_{\mathbf{k}}(t) d t \cdot \frac{1}{T} \int_{t_{0}}^{t_{\mathrm{f}}} \mathbf{v}_{\mathbf{k}}(t) d t\right) .
\end{aligned}
$$

These equations are analytically integrated using the right hand side of equations (6) and (7).

\subsubsection{Reaction rates}

Reaction rates are derived by two different methods. The first one is to calculate a rate $\nu_{i, j}$ for a process $j$ of the species $i$ with cross section $\sigma_{i, j}$ by the convolution

$$
\nu_{i, j}=k_{i} \sqrt{\frac{2}{m_{\mathrm{e}}}} \int_{0}^{\infty} \sigma_{\mathrm{j}}(\varepsilon) \varepsilon f(\varepsilon) \mathrm{d} \varepsilon .
$$

For such a numerical integration a precise EEDF is necessary with low statistical noise.

Another method to obtain a certain reaction rate is to count the events for the corresponding process. In section 2.5, we introduced two methods of computationally keeping the electron number $N_{\mathrm{e}}(t)$ fixed or not. If the number of electrons is fixed, $N_{\mathrm{e}}(t)=N_{\mathrm{e}}\left(t_{0}\right)$, then the number of counts $N_{j}$ of a process increases linearly in time, and the rate $\nu_{i, j}$ can be obtained from $N_{j}$ between times $t$ and $t_{0}$ by

$$
\nu_{i, j}^{t_{0}, t}=\frac{N_{j}(t)-N_{j}\left(t_{0}\right)}{N_{\mathrm{e}}\left(t_{0}\right)\left(t-t_{0}\right)} .
$$


In case of non-fixed electron number $N_{\mathrm{e}}(t)$ increases exponentially and the effective ionization rate is given by

$$
\nu_{\mathrm{eff}}^{t_{0}, t}=\frac{\log N_{\mathrm{e}}(t)-\log N\left(t_{0}\right)}{t-t_{0}} .
$$

The rates of the individual processes such as attachment, ionization or excitation are calculated by

$$
\nu_{i, j}^{t_{0}, t}=\frac{N_{j}(t)-N_{j}\left(t_{0}\right)}{N_{j}\left(t_{0}\right)} \frac{\nu_{\mathrm{eff}}}{e^{\nu_{\mathrm{eff}} t}-1} .
$$

The final results for the reaction rates are the averaged rates from equations (27-29) over the time interval $\left[t_{0}, t_{f}\right]$

$$
\nu_{i, j}=\left\langle\nu_{i, j}^{t_{0}, t}\right\rangle_{t_{0}}^{t_{\mathrm{f}}}, \nu_{\mathrm{eff}}=\left\langle\nu_{\mathrm{eff}}^{t_{0}, t}\right\rangle_{t_{0}}^{t_{\mathrm{f}}}
$$

\section{Testing and comparison with other programs}

\subsection{Model gases: Maxwell and Reid's Ramp model}

The Maxwell model [23] is given by an elastic cross section $\sigma_{\mathrm{el}}(\varepsilon)$ of the form $\sigma_{\mathrm{el}}(\varepsilon)=6 \cdot 10^{-20} \varepsilon^{-1 / 2} \mathrm{~m}^{2}$ ( $\varepsilon$ in eV), whereas Reid's Ramp model [24] is given by a constant elastic cross section $\sigma_{\mathrm{el}}(\varepsilon)=6 \cdot 10^{-20} \mathrm{~m}^{2}$ and a linearly increasing excitation cross section $\sigma_{\text {ex }}(\varepsilon)=10 \cdot \operatorname{Heaviside}(\varepsilon-0.2) \cdot 10^{-20} \mathrm{~m}^{2}$, with a threshold energy of $0.2 \mathrm{eV}$. The mass $M$ of the background gas is 4 atomic units and the temperature is equal to zero. We compare our results for the mean energy $\langle\varepsilon\rangle$, drift velocity in z-direction $w_{z}$ and density reduced diffusion constants $N D_{x}, N D_{y}$ and $N D_{z}$, see table 1 . The latter is always within the $3 \sigma$ error of our simulation data and the agreement is $\lesssim 0.1 \%$ for the mean energy and the drift velocity, and $\lesssim 1 \%$ for the diffusion constant.

\subsection{Benchmark data}

The calculations of benchmark data were done with BOLSIG+ (version 08.2012, default settings: Number of energy intervals: 100, Grid type: Automatic, Precision $10^{-9}$, Convergence $10^{-3}$, Iterations 100). Monte Carlo simulations were done with MAGBOLTZ (version 10.0.2, setting $4 \cdot 10^{8}$ real collisions). Both programs provide the flux drift velocity and the flux diffusion constant for isotropic electron scattering.

For the real gases argon Ar, nitrogen $\mathrm{N}_{2}$ and oxygen $\mathrm{O}_{2}$ Biagi's cross sections were chosen [30]. According to the documentation of the MAGBOLTZ 
Table 1: A comparison of the transport parameters for the Maxwell $(E / N=$ $1 \mathrm{Td})$ and Ramp model $(E / N=12 \mathrm{Td})$ using the present code with the results from [29]. The simulations stopped after $\sim 2 \cdot 10^{9}$ and $\sim 2 \cdot 10^{8}$ real collisions for the Maxwell and the Ramp model, respectively.

\begin{tabular}{llll}
\hline $\begin{array}{l}\langle\varepsilon\rangle \\
(\mathrm{eV})\end{array}$ & $\begin{array}{l}w_{z} \\
\left(10^{3} \mathrm{~ms}^{-1}\right)\end{array}$ & $\begin{array}{l}N D_{x, y} \\
\left(10^{24} \mathrm{~m}^{-1} \mathrm{~s}^{-1}\right)\end{array}$ & $\begin{array}{l}N D_{z} \\
\left(10^{24} \mathrm{~m}^{-1} \mathrm{~s}^{-1}\right)\end{array}$ \\
\hline Maxwell & & & \\
0.5058 & 4.946 & 1.663 & 1.685 \\
$0.5064^{a}$ & $4.943^{a}$ & $1.668^{a}$ & $1.668^{a}$ \\
\hline Reid & & & \\
0.270 & 6.851 & 1.133 & 0.566 \\
$0.269^{b}$ & $6.838^{b}$ & $1.135^{b}$ & $0.569^{b}$ \\
\hline
\end{tabular}

${ }^{a}$ Calculated values at zero temperature using the analytical expressions in $[29] ;{ }^{b}$ Values taken from table 3 in [29].

code the cross sections of $\mathrm{N}_{2}$ and $\mathrm{O}_{2}$ are equal in MAGOLBTZ-versions 10.0.2 and 8.9 and therefore direct comparison of our results with those of BOLSIG+ and MAGBOLTZ is possible. However, for the argon cross sections used in MAGBOLTZ-version 10.0.2 the documentation states: "update with small increase in excitation cross sections in resonance region for s-levels and compensating increase in elastic momentum transfer cross section at the maximum $(11 \mathrm{eV}) "$.

Furthermore, to show the applicability of our program for the case of gas mixtures as well as for strong attachment, we perform simulations in $\mathrm{N}_{2} / \mathrm{O}_{2}$ mixtures with the cross section sets from before, tetrafluoromethane $\mathrm{CF}_{4}$ [31] and sulfur hexafluoride [32]. No MAGBOLTZ simulations are performed for $\mathrm{CF}_{4}$ and $\mathrm{SF}_{6}$, since direct comparison with the same cross section input is not possible.

\subsection{Simulation settings}

Different simulation settings are tested in argon, namely the treatment of non-conservative collisions in terms of fixed and non-fixed electron numbers, different electron numbers $N_{\mathrm{e}}$, pressures $p$ and energy sharings $W$. The gas particle number density $N$ is deduced from the pressure $p$ and the temperature $T=300 \mathrm{~K}$ via the ideal gas law. The results for $\langle\varepsilon\rangle, \tilde{w}, N \tilde{D}_{z}$ and $\nu_{\text {eff }} / N$ in argon are presented in figure 3 . For all parameters, the difference 
between the results of the fixed and non-fixed method is equal besides statistical noise. The standard deviation $\sigma$ of all parameters derived from the non-fixed method increases with increasing $E / N$ due to the limited statistics as mentioned in section 2.5. Thus, using the method of a fixed electron number seems to be valid up to high electron multiplication. We also verified this method for the case of strong attachment in $\mathrm{CF}_{4}$, which is not shown here. There is no significant influence of $p$ and $N_{\mathrm{e}}$ on the results, whereas the energy sharing $W$ has a significant influence on $\tilde{w}$ and $N \tilde{D}$ towards high $E / N$-values.

For the remaining simulations, the following settings were used: fixed $N_{\mathrm{e}}(t)=10^{4}, p=10^{5} \mathrm{~Pa}, \mathrm{~W}=0.5$. For the calculation of rates from the EEDF according to equation (26) the number of energy intervals was set to 2000. For these settings, figure 4 displays the EEPF in argon for a low and a high $E / N$ value in comparison to the EEPF from BOLSIG+.

\subsection{Accuracy}

The accuracy of the simulation results increases with the number of real collisions and thus with the computation time. Figure 5 shows the relative error of $\langle\varepsilon\rangle, w, \tilde{w}, N D_{z}, N \tilde{D}_{z}$ and $\nu_{\text {eff }}$ as a function of the number of real collisions. The relative errors of $\tilde{w}$ and $N \tilde{D}_{z}$ are limited to $\gtrsim 0.1 \%$ and $\gtrsim 1 \%$ respectively, whereas bulk data, rates and mean energy become more accurate as the number of collisions increases. The accuracy of the EEDF can be crossed-checked by comparing the effective ionization rates calculated by the two independent methods described in section 2.6.3.

\subsection{Real gases: $\mathrm{Ar}, \mathrm{N}_{2}, \mathrm{O}_{2}$ and $\mathrm{N}_{2} / \mathrm{O}_{2}$ mixtures}

In this section, we compare the results of our simulation code with those of BOLSIG + and MAGBOLTZ for pure argon, $\mathrm{N}_{2}$ and $\mathrm{O}_{2}$ and $\mathrm{N}_{2} / \mathrm{O}_{2}$ mixtures with the cross section data given in section 3.2.

For the pure gases, our results for the mean energy, drift velocity and effective ionization rate constant as a function of $E / N$ are presented in figure 6 and the results for the reduced diffusion constant are shown in figure 7 . The energy data and transport coefficients are derived following section 2.6.1 and section 2.6.2. The effective ionization rate constant $\nu_{\text {eff }} / N$ is calculated by numerical integration of the EEDF using equation (26), as well as by counting ionization/attachment events and using equation (27). In general, we find good agreement between our results for $\langle\varepsilon\rangle, \tilde{w}$ and $\nu_{\text {eff }} / N$ and the results from BOLSIG + and MAGBOLTZ for the considered $E / N$-range, see 

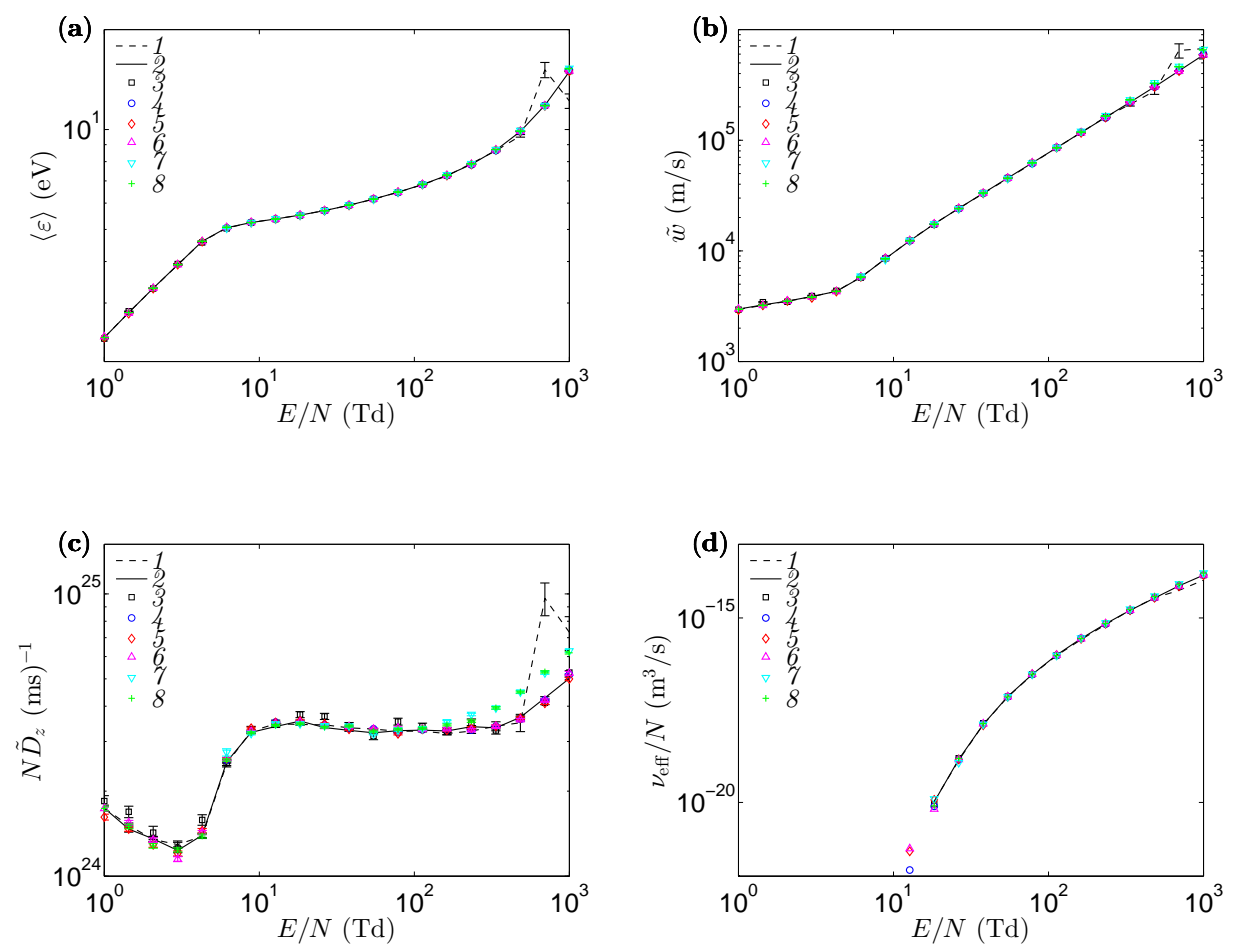

Figure 3: Results in argon as a function of the reduced field $E / N$ for (a) mean electron energy $\langle\varepsilon\rangle$, (b) flux drift velocity $\tilde{w}$, (c) reduced longitudinal flux diffusion constant $N \tilde{D}_{z}$ and (d) effective ionization rate constant $\nu_{\text {eff }} / N$ obtained by counting. Shown are the results for 8 different numerical settings for electron number, pressure and energy sharing $\left(N_{\mathrm{e}}, p, W\right)$ : 1 (nonfixed, $\left.10^{5}, 0.5\right), 2\left(10^{4}, 10^{5}, 0.5\right), 3\left(10^{3}, 10^{5}, 0.5\right), 4\left(10^{4}, 10^{5}, 0.5\right), 5$ $\left(10^{4}, 1,0.5\right), 6\left(10^{4}, 10^{3}, 0.5\right), 7\left(10^{4}, 10^{5}, 0\right)$ and $8\left(10^{4}, 10^{5}, 1\right)$ 


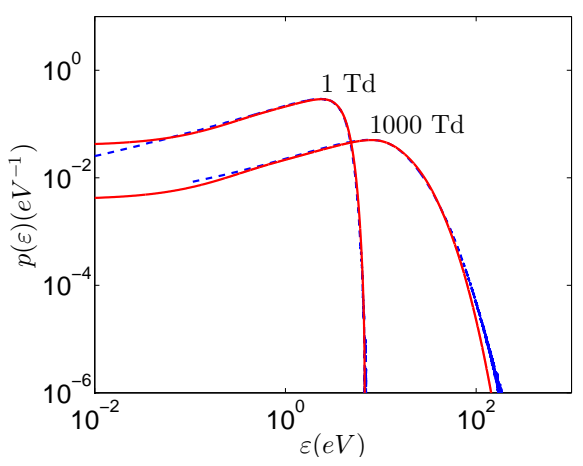

Figure 4: Present results in pure argon for the electron energy probability function (EEPF) for $E / N=1 \mathrm{Td}$ and $E / N=1000 \mathrm{Td}$ (dashed line). Settings: fixed $N_{\mathrm{e}}(t)=10^{4}, p=10^{5} \mathrm{~Pa}, \mathrm{~W}=0.5$. BOLSIG+ (full line).

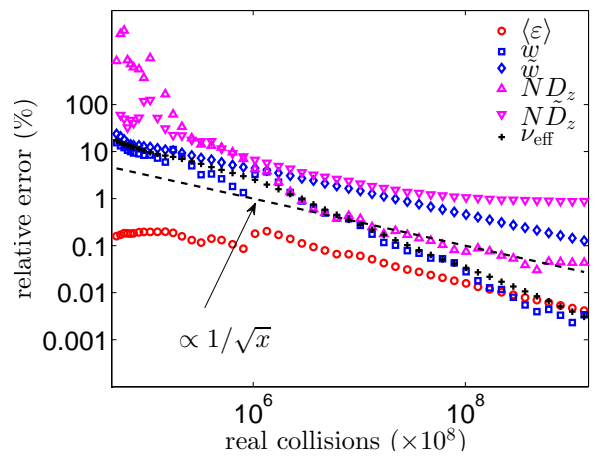

Figure 5: Relative error of transport data and reaction rates vs number of real collisions. Settings: pure argon, $E / N=100 \mathrm{Td}$, fixed $N_{\mathrm{e}}(t)=10^{4}$, $p=10^{5} \mathrm{~Pa}, \mathrm{~W}=0.5$. For comparison: the $1 / \sqrt{x}$ tendency $(x=$ number of real collisions) is indicated with the dashed line. 

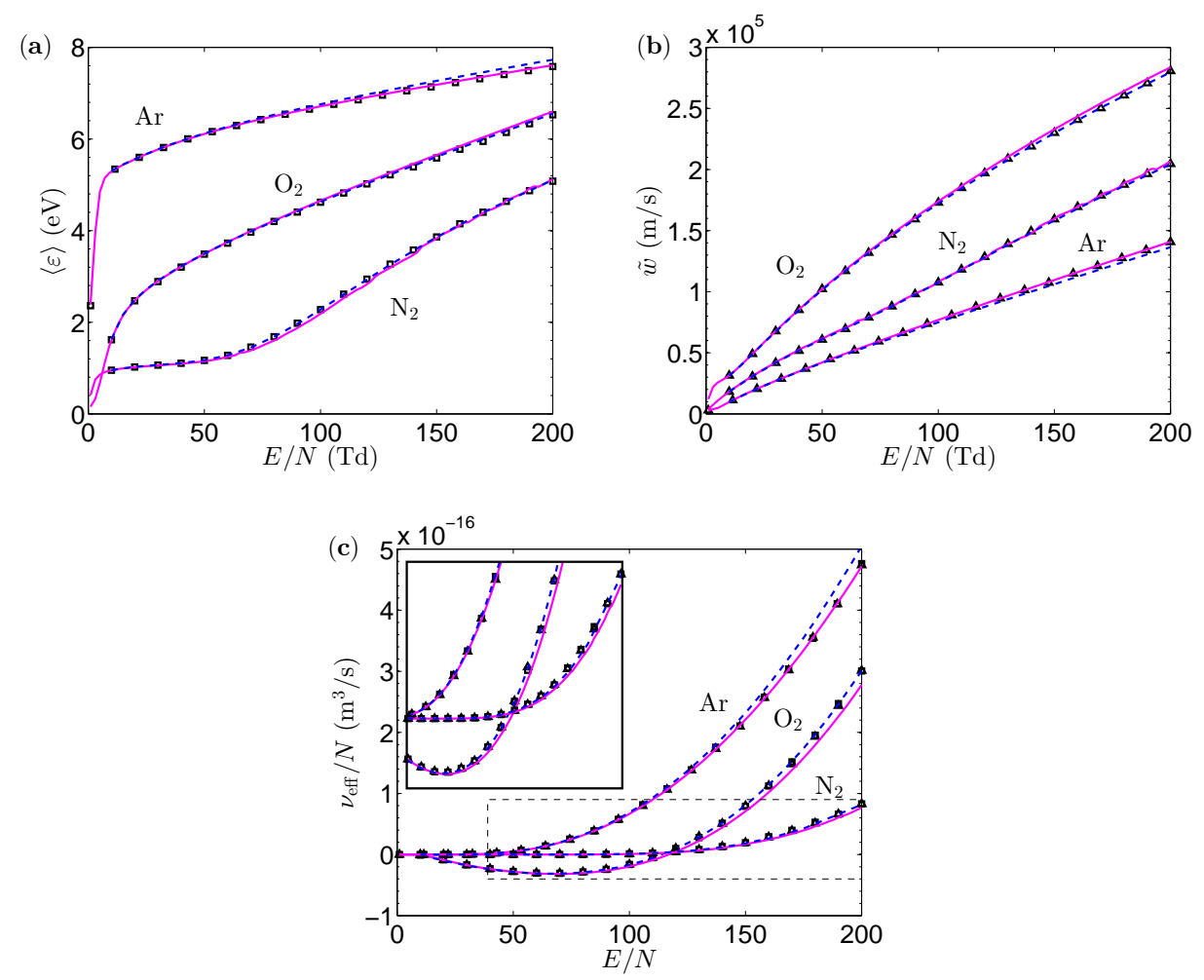

Figure 6: Present results in pure $\mathrm{Ar}, \mathrm{N}_{2}$ and $\mathrm{O}_{2}$ as a function of the reduced field $E / N$ for (a) mean electron energy $\langle\varepsilon\rangle(\square)$, (b) flux drift velocity $\tilde{w}(\triangle)$ and (c) effective ionization rate constant $\nu_{\text {eff }} / N$ obtained by counting $(\square)$ and convolution $(\triangle)$. BOLSIG + results (solid line), MAGBOLTZ results (dashed line).

table 2. The agreement for $\mathrm{N}_{2}$ and $\mathrm{O}_{2}$ with MAGBOLTZ is better than with BOLSIG + . The slight deviations from MAGBOLTZ results for argon might be related to the update in the cross section set as described in section 3.2. For the diffusion constant our agreement with BOLSIG + is poor, whereas we find good agreement with MAGBOLTZ. The present results for $D_{x, y}$ and $D_{z}$ are in general smaller than the BOLSIG + results. The inaccuracy of $D_{x, y}$ and $D_{z}$ calculated by means of two-term approximation is well-known $[19,33]$. Figure 8 shows $\tilde{w}$ and $\nu_{\text {eff }} / N$ for the $\mathrm{N}_{2} / \mathrm{O}_{2}$ mixtures $100 / 0 \%$, $80 / 20 \%, 60 / 40 \%, 40 / 60 \%, 20 / 80 \%$ and $0 / 100 \%$. The discrepancy between our results and those from BOLSIG+ and MAGBOLTZ is of the same order 

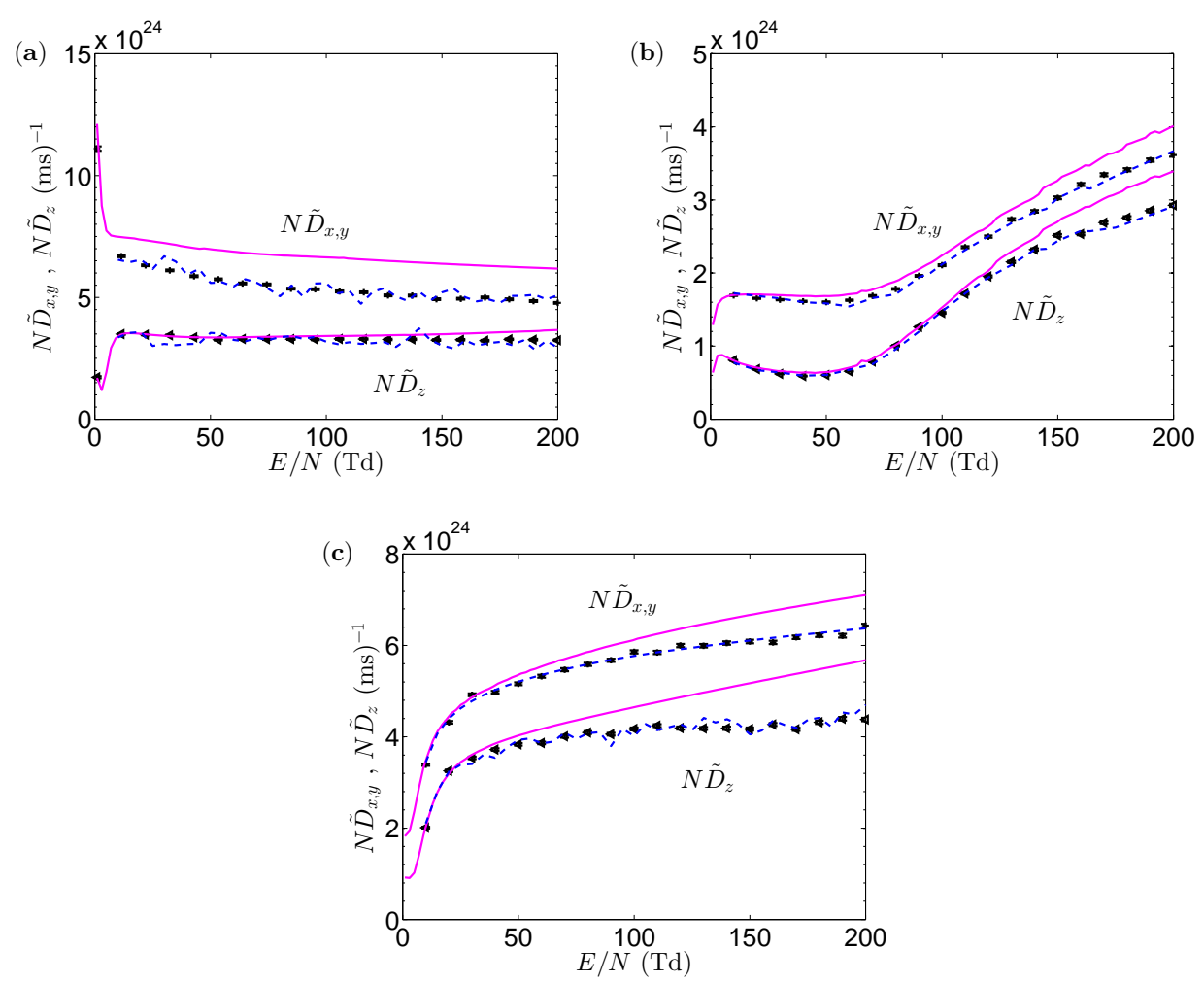

Figure 7: Reduced flux diffusion constant $N \tilde{D}$ (longitudinal: $\triangleleft$, transversal:+) vs reduced field $E / N$ in (a) $\mathrm{Ar}$, (b) $\mathrm{N}_{2}$ and (c) $\mathrm{O}_{2}$. BOLSIG + results (solid line), MAGBOLTZ results (dashed line). 

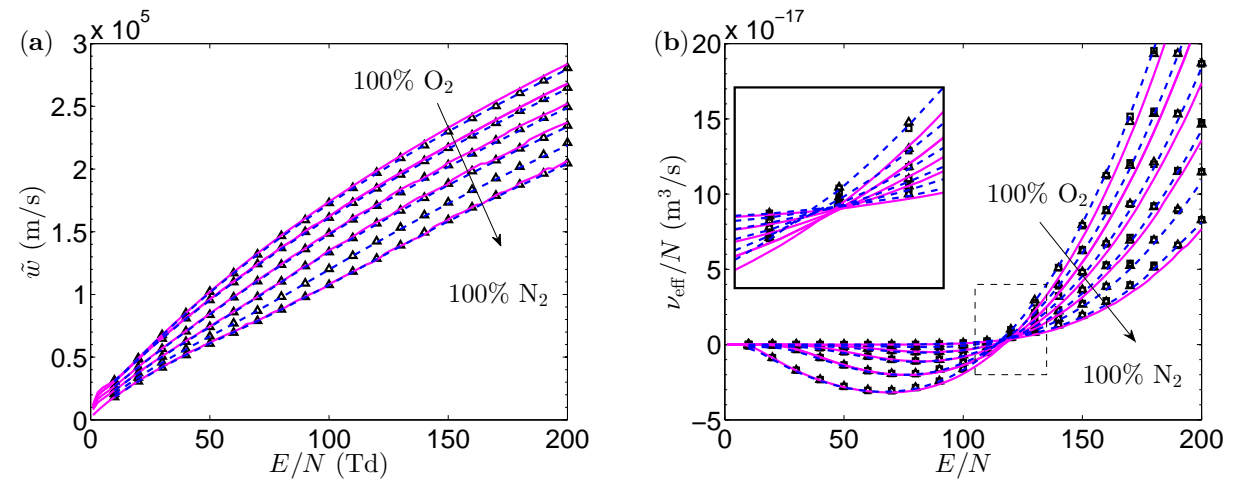

Figure 8: Present results in $\mathrm{N}_{2} / \mathrm{O}_{2}$ mixtures in the ratios of $100 / 0 \%, 80 / 20 \%$, $60 / 40 \%, 40 / 60 \%, 20 / 80 \%$ and $0 / 100 \%$ vs reduced field $E / N$ for (a) flux drift velocity $\tilde{w}(\triangle)$ and (b) effective reaction rate constant $\nu_{\text {eff }} / N$ obtained by counting $(\square)$ and convolution $(\triangle)$. BOLSIG + results (solid line), MAGBOLTZ results (dashed line).

as for the pure gases.

\subsection{Strongly attaching gases: $\mathrm{CF}_{4}$ and $S F_{6}$}

For the gases $\mathrm{CF}_{4}$ and $\mathrm{SF}_{6}$ we calculate the mean energy, transport coefficients, effective $\nu_{\text {eff }} / N$, ionization $\nu_{\mathrm{i}} / N$ and attachment rate constants $\nu_{\mathrm{a}} / N$. The cross section set used for $\mathrm{SF}_{6}$ contains the effective momentum transfer cross section from which the elastic momentum transfer cross section is calculated as described in section 2.1.

Figure 9 displays METHES and BOLSIG+ results for $\mathrm{CF}_{4}$. The agreement with the BOLSIG+ results is good for $\langle\varepsilon\rangle, \nu_{\text {eff }} / N, \nu_{\mathrm{i}} / N, \nu_{\mathrm{a}} / N$ and $\tilde{w}$, see also table 2 . For the lowest $E / N$-value our results for $\tilde{w}$ are significantly smaller than BOLSIG + results. Our results for $N \tilde{D}$ are in general smaller compared to the BOLSIG + results. This discrepancy is due to the limited applicability of the two-term approximation in case of a pronounced Ramsauer Townsend minimum of the elastic cross section compared to the inelastic cross sections, see e.g. [19, 20].

Figure 10 shows METHES and BOLSIG+ results for $\mathrm{SF}_{6}$. For $E / N>$ $20 \mathrm{Td}$, the results for $\langle\varepsilon\rangle, \tilde{w}, N \tilde{D}$ and the reaction rates agree with BOLSIG+ within the numbers given in table 2 . For $E / N<10 \mathrm{Td}$, our results for $\nu_{\mathrm{i}} / N$ and $N \tilde{D}$ show large $(>100 \%)$ deviations from BOLSIG+. In addition, the 

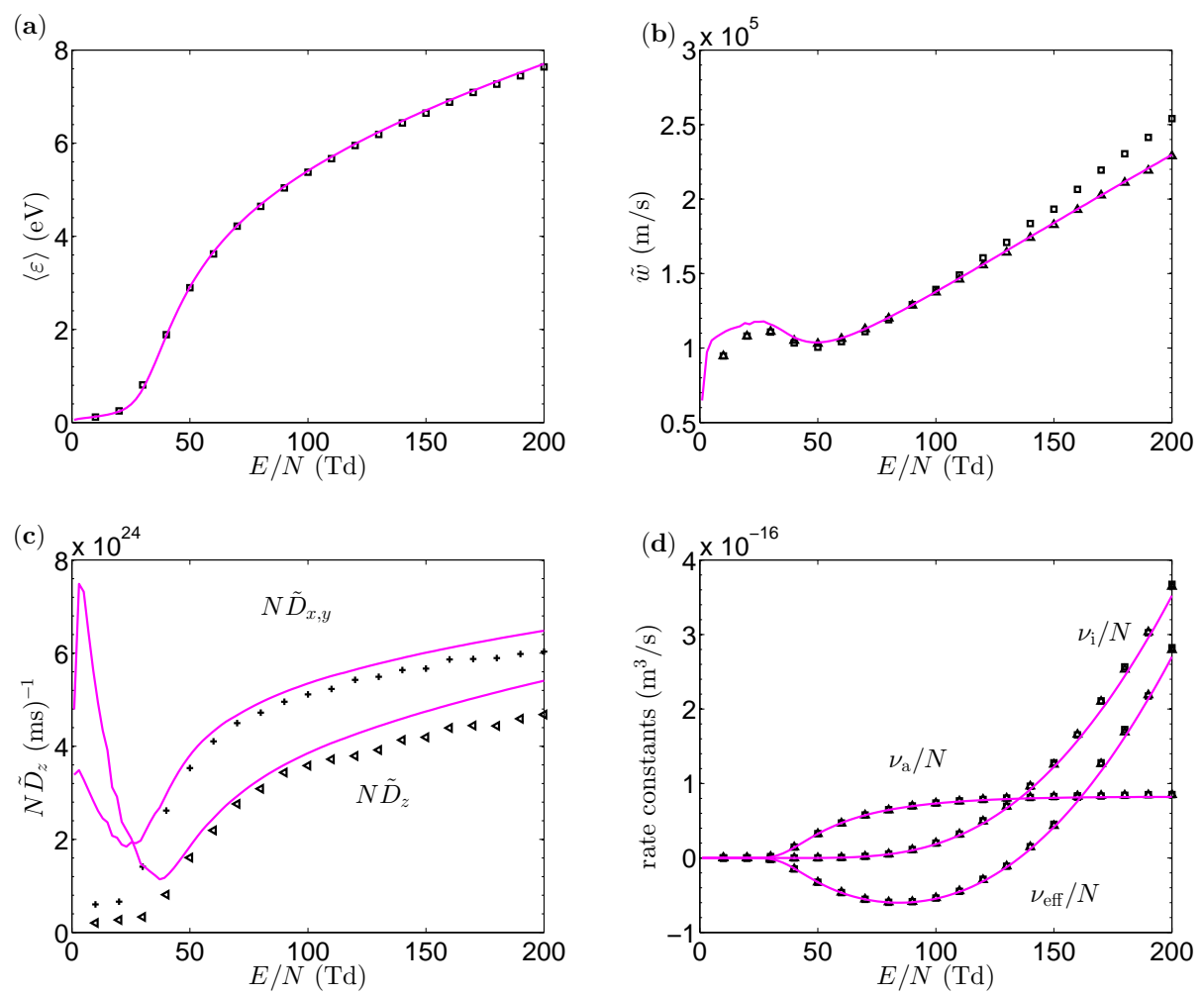

Figure 9: Present results in $\mathrm{CF}_{4}$ as a function of the reduced field $E / N$ for (a) mean electron energy $\langle\varepsilon\rangle(\square)$, (b) bulk $w(\square)$ and flux $\tilde{w}(\triangle)$ drift velocity, (c) reduced flux diffusion constant $N \tilde{D}$ (longitudinal: $\triangleleft$, transversal: + ) and (d) effective $\nu_{\text {eff }} / N$, ionization $\nu_{\mathrm{i}} / N$ and attachment rate constant $\nu_{\mathrm{a}} / N$ obtained by counting $(\square)$ and convolution $(\triangle)$. BOLSIG + results (solid line). 


\begin{tabular}{ccccc}
\hline & $\langle\varepsilon\rangle$ & $\tilde{w}$ & $N \tilde{D}$ & $\nu_{\text {eff }} / N$ \\
\hline $\mathrm{Ar}$ & $0.4 / 2$ & $0.1 / 3$ & $30 / 5$ & $0.4 / 7$ \\
$\mathrm{~N}_{2}$ & $5 / 0.5$ & $1 / 1$ & $15 / 3$ & $7 / 1.5$ \\
$\mathrm{O}_{2}$ & $1 / 0.5$ & $1 / 0.3$ & $30 / 7$ & $8 / 0.1$ \\
$\mathrm{CF}_{4}$ & $0.8 /-$ & $15 /-$ & $>100 /-$ & $3 /-$ \\
$\mathrm{SF}_{6}$ & $30 /-$ & $20 /-$ & $75 /-$ & $20 /-$ \\
\hline
\end{tabular}

Table 2: Relative difference in \% between results from MEHTES and BOLSIG+/MAGBOLTZ for selected quantities. The listed values are associated with those data points from figure $6,7,9$ and 10 that reveal the largest discrepancies between METHES and BOLSIG+ and between METHES and MAGBOLTZ. The numbers for $\mathrm{SF}_{6}$ exclude the noisy data points from BOL$\mathrm{SIG}+$ simulations.

"jump" of the mean energy, transport data and $\nu_{\mathrm{a}} / N$ at $E / N \approx 10 \mathrm{Td}$ is shifted to higher $E / N$-values compared to the BOLSIG+ data. For this regime, the reliability of the BOLSIG + is questionable due to numerical noise of $\langle\varepsilon\rangle, N \tilde{D}$ and the reaction rates over several orders of magnitude.

For both attaching gases, flux and bulk transport data are equal in the absence of non-conservative collisions. However, when attachment becomes dominant, flux and bulk data differ strongly. This is significant for the drift velocities in $\mathrm{CF}_{4}$ and $\mathrm{SF}_{6}$ over the whole $E / N$-range.

\section{Conclusion}

A simulation program for the electron transport in the presence of uniform electric fields has been developed using a Monte Carlo technique. The program uses the cross section sets that can be downloaded from the LXCat database (or comply with this format) in order to calculate transport data, reaction rates and the electron energy distribution. The code was described in detail and applied to various gases to test the different cases of strong ionization, strong attachment and gas mixtures. Results were compared to the literature, BOLSIG+ or MAGBOLTZ. Good agreement was found with BOLSIG+ and excellent agreement with MAGBOLTZ, when the same cross section inputs were used. Only for the diffusion constant, (expected) large discrepancies to BOLSIG + were seen. The visualization of the non-steady-state evolution of an electron swarm is directly possible. For the experienced user, changes or improvements of the code are possible due 

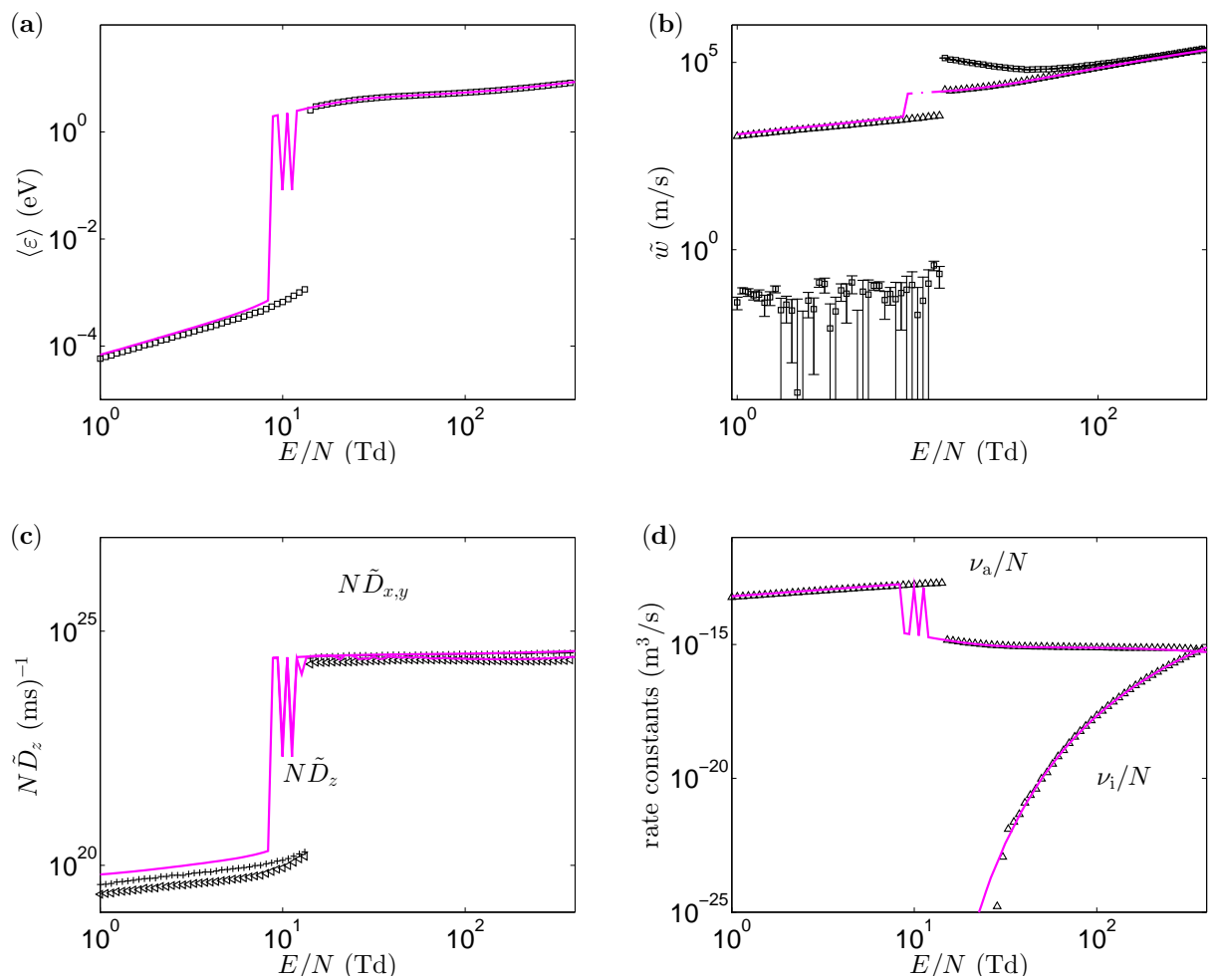

Figure 10: Present results in $\mathrm{SF}_{6}$ as a function of the reduced field $E / N$ for (a) mean electron energy $\langle\varepsilon\rangle(\square)$, (b) bulk $w(\square)$ and flux $\tilde{w}(\triangle)$ drift velocity, (c) reduced flux diffusion constant $N \tilde{D}$ (longitudinal: $\triangleleft$, transversal: + ) and (d) ionization $\nu_{\mathrm{i}} / N$ and attachment rate constant $\nu_{\mathrm{a}} / N$ obtained by convolution $(\triangle)$. BOLSIG+ results (solid line). 
to the object-orientated structure of the code and its documentation. The treatment of space- and time-dependent fields requires modifications of the program, in particular for the null-collision technique and the integration of the electron trajectories. These code modifications will be targeted in the future. The present code has the name METHES and can be downloaded from http://www.lxcat.net/download/METHES/.

\section{Acknowledgement}

We thank S. Dujko for valuable discussions and S. Pancheshnyi for support with uploading the code to LXCat. This work is financially supported by ALSTOM Grid GmbH, Pfiffner AG and ABB Switzerland.

\section{References}

[1] S. F. Biagi, Nucl. Instr. and Meth. A 421 (1999) 234-240.

[2] D. Bosnjakovic, Z. Lj. Petrovic, R. D. White, S. Dujko, J. Phys. D: Appl. Phys. 47 (2014) 435203.

[3] L. G. Christophorou, J. K. Olthoff, Applied Surface Science 192 (2002) 309.

[4] L. G. Christophorou, Nucl. Instr. and Meth. A 268 (1988) 424-433.

[5] K. Kumar, Physics reports 112 (1984) 319-375.

[6] R. E. Robson, K. F. Ness, Phys. Rev. A 33 (1986) 2068.

[7] G. J. M. Hagelaar, L. C. Pitchford, Plasma Sources Sci. Technol. 14 (2005) 722-733.

[8] H. R. Skullerud, J. Phys. D: Appl. Phys. 1 (1969) 1567.

[9] G. W. Fraser, E. Mathieson, Nucl. Instr. and Meth. A 247 (1986) 544 565.

[10] M. Yousfi, A. Hennad, A. Alkaa, Phys. Rev. E 49 (1994) 3264.

[11] V. Vahedi, M. Surendra, Comp. Phys. Commun. 87 (1995) 179-198. 
[12] S. Dujko, R. D. White, Z. Lj. Petrovic, J. Phys. D: Appl. Phys. 41 (2008) 245205.

[13] R. E. Robson, R. D. White, Z. Lj. Petrovic, Rev. Mod. Phys. 77 (2005) 1303.

[14] W. M. Huo, F. A. Gianturco, Computational Methods for ElectronMolecule Collisions, Plenum, New York.

[15] L. G. Christophorou (Ed.), Electron-molecule interactions and their applications Vol. 1, Academic Press.

[16] L. G. H. Huxley, R. W. Crompton, The diffusion and drift of electrons in gases, Wiley, New York.

[17] S. Pancheshnyi, S. Biagi, M. C. Bordage, G. J. M. Hagelaar, W. L. Morgan, A. V. Phelps, L. C. Pitchford, Chem. Phys. 398 (2012) 148.

[18] R. D. White, R. E. Robson, B. Schmidt, M. A. Morrison, J. Phys. D: Appl. Phys. 36 (2003) 3125.

[19] Z. Lj. Petrovic, S. Dujko, D. Maric, G. Malovic, Z. Nikitovic, O. Sasiic, J. Jovanovic, V. Stojanovic, M. Radmilovic-Radenovic, J. Phys. D: Appl. Phys. 42 (2009) 194002.

[20] M.-C. Bordage , P. Segur, L. G. Christophorou, J. K. Olthoff, J. Phys. D: Appl. Phys. 86 (1999) 3558.

[21] R. E. Robson, Aust. J. Phys. 44 (1991) 685692.

[22] D. Bosnjakovic, Z. Lj. Petrovic, R. D. White, S. Dujko, J. Phys. D: Appl. Phys. 47 (2014) 435203.

[23] S. Chapman, T. G. Cowling, The Mathematical Theory of Non-uniform Gases, Cambridge, London, 1935.

[24] I. D. Reid, Aust. J. Phys. 32 (1979) 231.

[25] J. Itoh, T. Musha, J. Phys. Soc. Jpn. 15 (1960) 1675-1680.

[26] S. Dujko, R. D. White, Z. Lj. Petrovic, R. E. Robson, Phys. Rev. E 81 (2010) 046403. 
[27] T. Taniguchi, H. Tagashira, Y. Sakai, J. Phys. D: Appl. Phys. 10 (1977) 2301.

[28] Z. Lj. Petrovic, Z. M. Raspopovic, S. Dujko, T. Makabe, Appl. Surf. Sci. 192 (2002) 1-25.

[29] K. F. Ness, J. Phys. D: Appl. Phys. 27 (1994) 1848-1861.

[30] Biagi-v8.9 database, www.lxcat.net, retrieved on June 112015.

[31] Hayashi database, www.lxcat.net, retrieved on June 112015.

[32] Phelps database, www.lxcat.net, retrieved on June 112015.

[33] K. F. Ness, R. E. Robson, Phys. Rev. A 34 (1986) 2185-2209. 